\title{
The Mercator telescope: relevance, status and future
}

\author{
Gert Raskin $^{a}$, Wim Pessemier ${ }^{a}$, Florian Merges ${ }^{b}$, Jesus Pérez Padilla ${ }^{b}$, Saskia Prins ${ }^{b}$ and Hans \\ Van Winckel ${ }^{a}$ \\ ${ }^{a}$ Institute of Astronomy, KU Leuven, Celestijnenlaan 200D, B-3001 Leuven, Belgium \\ ${ }^{b}$ Mercator Telescope, Roque de Los Muchachos Observatory, La Palma, Spain
}

\begin{abstract}
In todays era of ever growing telescope apertures, there remains a specific niche for meter-class telescopes, provided they are equipped with efficient and dedicated instruments. In case these telescopes have permanent and long-term availability, they turn out very useful for intensive monitoring campaigns over a large range of time-scales. Flexible scheduling and time allocation allow small telescopes to rapidly seize new opportunities or provide immediate follow-up observations to complement data from large ground-based or space-borne facilities. The Mercator telescope, a 1.2-m telescope, installed at the Roque de Los Muchachos Observatory on La Palma (Canary Islands, Spain), successfully targets this niche of intensive monitoring and flexible scheduling. Mercator is already in operation since 2001 and has seen several upgrades in the mean time. In this contribution we give an update about the actual telescope status and its performance. We also present the Mercator instrument suite that currently consists of two instruments. The workhorse instrument is HERMES, a very efficient and stable fibre-fed high-resolution spectrograph. Recently, the MAIA imager was commissioned. This is a threechannel photometric instrument that observes a large field simultaneously in the different color bands. The MAIA detectors are unique $6 \mathrm{k} \times 2 \mathrm{k}$ frame transfer devices which also allow for fast and continuous monitoring of variable phenomena.We discuss two important upcoming upgrades: a long-awaited automatic mirror cover and, more importantly, an entirely new telescope control system (TCS). This TCS is based on modern PLC technology, and relies on OPC UA and EtherCAT communication. Only commercially off-the-shelve hardware will be used for controlling the telescope. As a test case and as a precursor of the full TCS, such PLC systems are already deployed at Mercator to steer the Nasmyth mirror mechanism and to control the MAIA instrument. Finally, we also give an overview of the exploitation scheme of the telescope, the scheduling software that we developed to guarantee that time series or time-critical observations can be acquired in an efficient way, and how this all serves the most important research themes for Mercator, mainly in the domain of stellar astrophysics.
\end{abstract}

Keywords: telescope, astronomical instrumentation: spectroscopy, astronomical instrumentation: photometry, telescope control, PLC, OPC UA

\section{INTRODUCTION}

With extremely large telescopes (ELT's) dawning at the horizon, we are experiencing exiting times for the development of astronomical instrumentation. It is clear that these ELT's will have an enormous impact on observational astronomy and that they will open up completely new parameter spaces. However, we are convinced that, even with a collecting area that is a thousand times smaller, meter-class telescopes still occupy an important niche, full of relevant observations and valuable science. This is also the case for the 1.2-m Mercator telescope (www.mercator.iac.es [1]), installed at the Roque de Los Muchachos Observatory on La Palma (Canary Islands, Spain) and presented in section 2 of this contribution.

Mercator is a Belgian telescope, funded by the Flemish Community of Belgium and operated by the Institute of Astronomy of the University of Leuven (KU Leuven, Belgium). The main asset of the Mercator telescope is its permanent long-term availability. For the observations of variable astrophysical phenomena, the sampling frequency is in many cases more relevant than the collecting power of the telescope. Mercator allows our astronomers to embark on intensive monitoring campaigns that cover an extremely broad range of time scales, extending from a few nights up to several years. Furthermore, flexible time allocation and scheduling allow us

Further author information: gert.raskin@ster.kuleuven.be 
to quickly seize emerging opportunities and obtain immediate follow-up observations to complement interesting data from much larger or space borne observing facilities. For obvious reasons, both flexibility and long-term availability are very difficult to attain from large telescopes.

Even more so than with a large telescope, it is important to dispose of efficient and dedicated instruments in order to do relevant and competitive science with a small telescope. Fortunately, obtaining a high-performance instrument suite for a small telescope is also much easier. Instrument design for small telescopes usually suffers much less from the broad variety of constraints that tends to accompany large facility projects serving a broad community. Efficiency and performance can only benefit from these reduced design constraints. In this respect, but also to allow sufficient space for intensive monitoring, we limited currently the instrument suite of Mercator to only two instruments, dedicated to high-resolution spectroscopy and to fast-cadence three-colour photometry.

In the rest of this contribution, we give a description of the telescope and the attached instruments. We also present the upgrades of the installation that are currently under development. Finally, we illustrate how a small telescope like Mercator is still capable of producing very important and unique science results.

\section{THE MERCATOR TELESCOPE}

Mercator is an alt-azimuthally mounted telescope with $f / 12$ Ritchey-Chrétien optics and a primary mirror diameter of $1.2 \mathrm{~m}$ (Fig. 1). Originally, the telescope was only equipped with two focal stations: Cassegrain and one Nasmyth focus. Since the installation of a rotation mechanism for M3 in 2012 [2], two Nasmyth focal stations became available, as well as two small folded Cassegrain foci. We built this telescope, a copy of the Swiss Euler telescope at the La Silla Observatory (Chile), in collaboration with the Observatoire de Genève (Switzerland). It was constructed in 2000 and is successfully in operation since early 2001. Two important upgrades of the facility are now nearing completion: an automatic mirror cover and, more importantly, a completely new telescope control system.

\subsection{Mirror cover}

Originally, the Mercator telescope was not equipped with a cover for the primary mirror. To protect the telescope optics when no observations take place, especially during periods of Calima (dust from the Sahara dessert) or high humidity, we now cover the telescope tube manually with a hemispheric cap. Handling the 1.5-m diamater cap is rather cumbersome and can also involve some risks. Therefore, we are now constructing an automatic telescope cover mechanism. The installation is planned for July 2014. The cover consists of 8 triangular petals, mounted at the top of the telescope tube (Fig. 2). These petals rotate 4 by 4 over $260^{\circ}$ between open and close. Each petal is driven by a torque limiter, a large reduction gear head and a DC motor. An absolute encoder on the shaft of each petal continuously monitors the position.

\subsection{Telescope control system}

The telescope is still controlled by an out-dated telescope control system (TCS), based on obsolete transputer technology. Currently, we are in the process of upgrading the TCS to a completely new system, fully based on programmable-logic-controller (PLC) technology [3]. Herewith, we rely as much as possible on industrial hardand software. OPC-UA, the emerging follow-up Unified Architecture standard of the original OPC (Object Linking and Embedding for Process Control), is used as communication technology to link all the components of the TCS. Compared to the traditional approach of a VMEbus system or Linux PC with custom-made control software and a variety of I/O hardware, this industrial solution increases reliability, facilitates maintenance and strongly reduces the development effort.

We selected PC-based controllers (so-called "soft-PLCs") from Beckhoff as our main development platform. Their key advantage is their versatility: a single controller can mix real-time applications (involving logic, motion, safety and even $\mathrm{C}++$ code) with non-real-time applications (such as communication servers and graphical interfaces). Since each PLC can hosts its own OPC UA server, and all our high-level TCS software components have an integrated OPC UA client, an excellent vertical integration is achieved. The same technology choice also supports horizontal integration, as PLCs can act as OPC UA clients. This means that they can interact with 


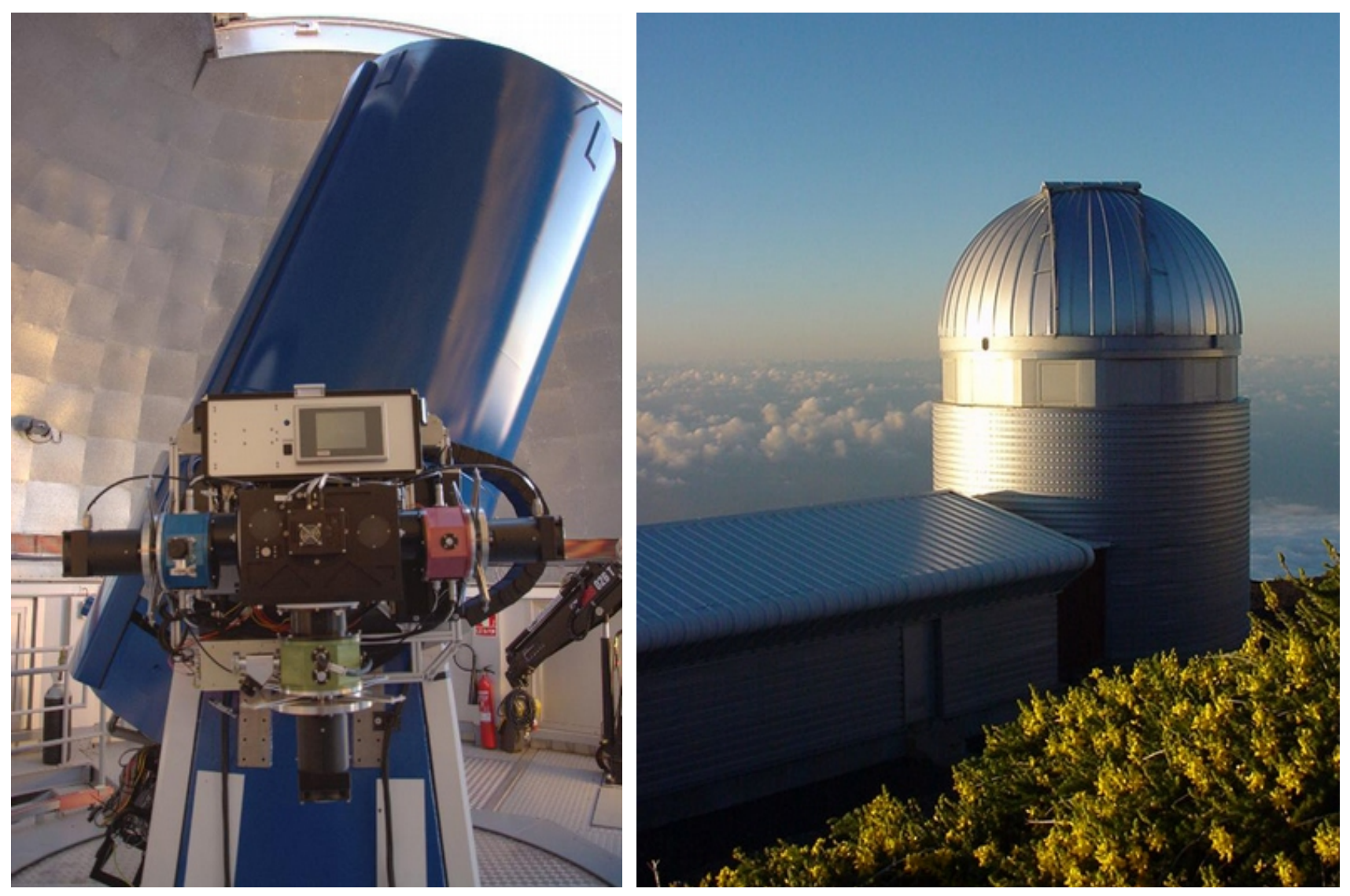

Figure 1. Mercator telescope with the MAIA imager at the Nasmyth focus (left), and telescope dome and building (right).
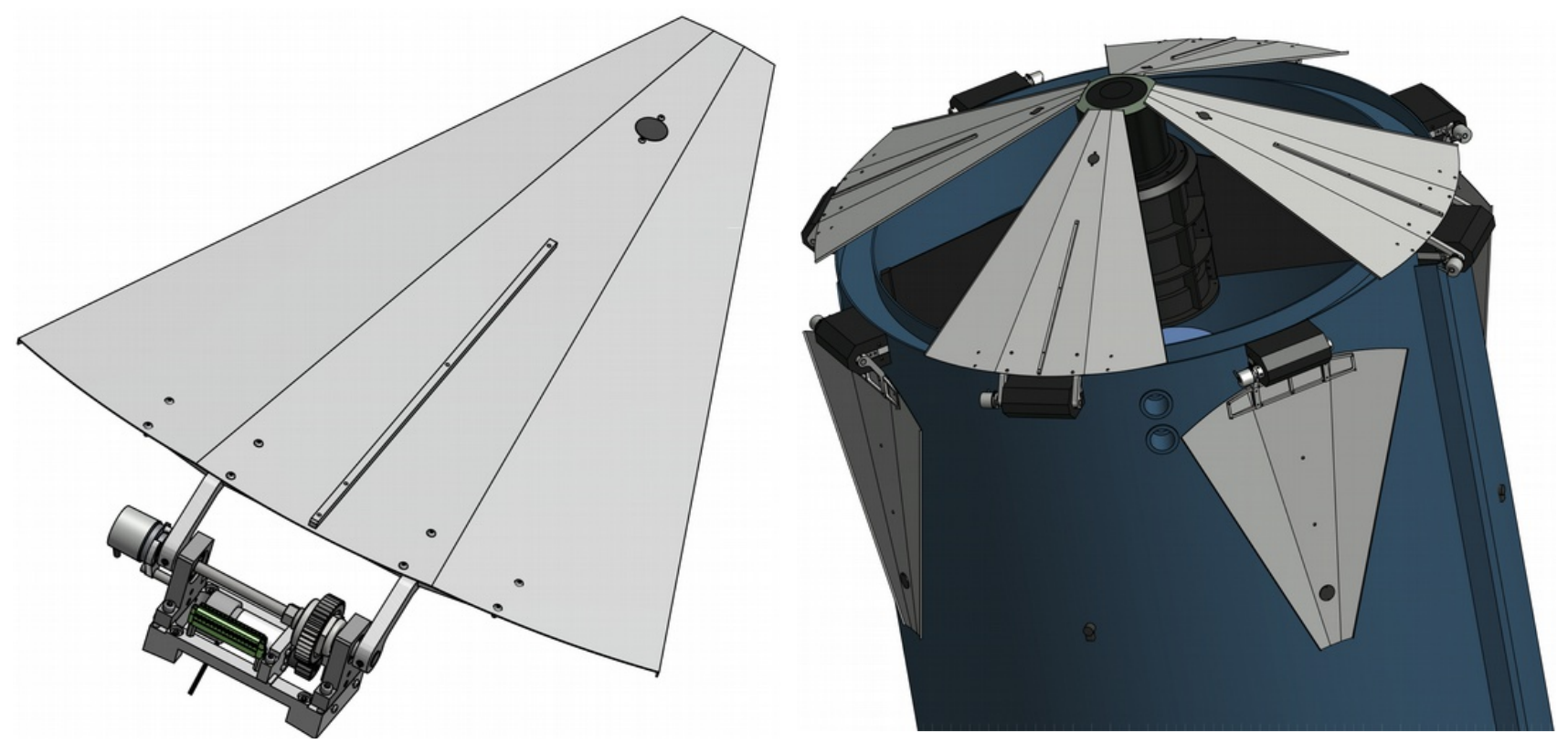

Figure 2. Telescope tube cover: single petal mechanism (left), and top of telescope tube with 4 petals in open and 4 petals in close position (right). 


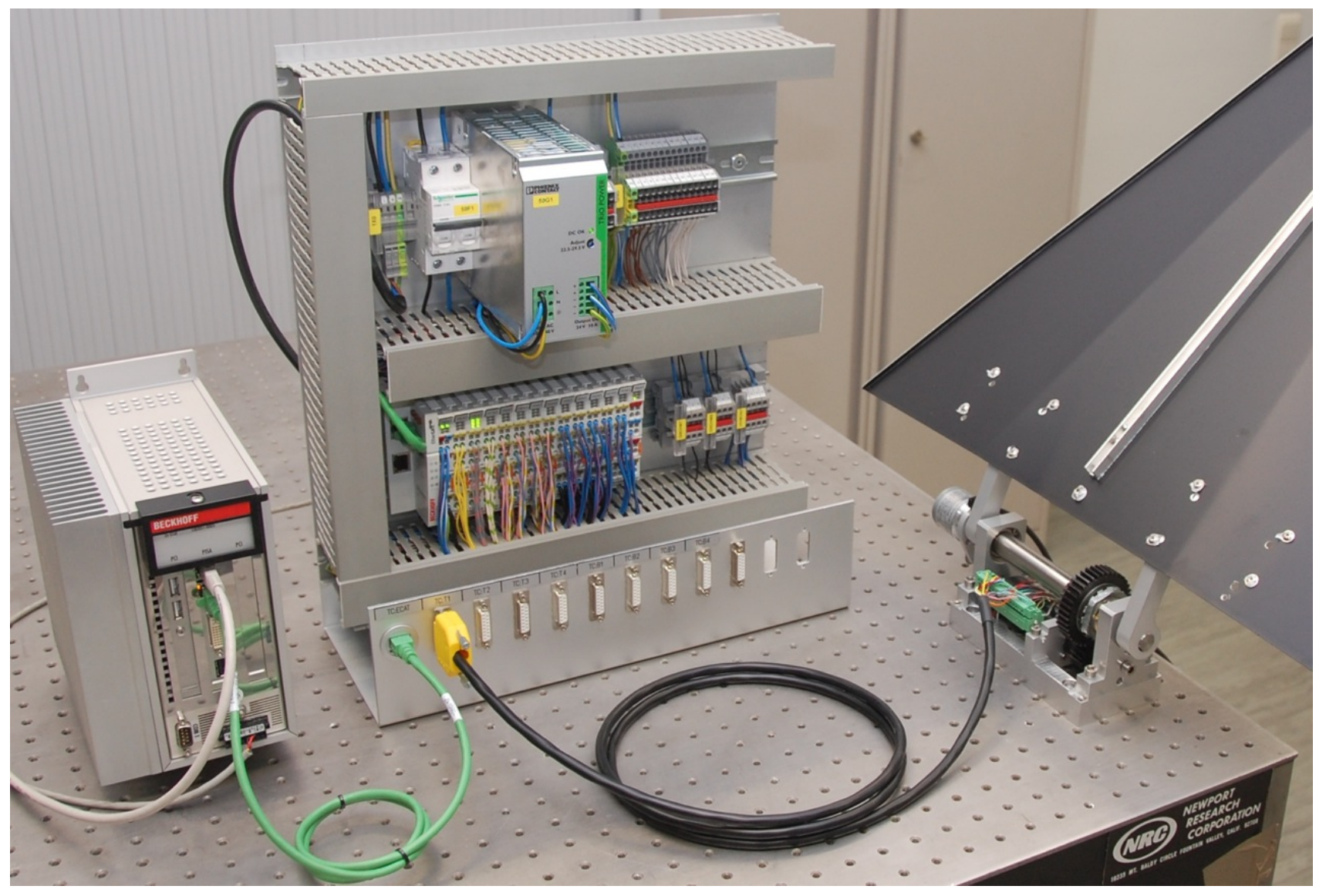

Figure 3. Picture of the control panel (centre) for the telescope cover mechanism (right). It includes I/O for reading 8 absolute SSI encoders, driving 8 DC motors and 8 holding magnets. It is linked to the PLC (left) through EtherCAT (green cable).

other controllers, or even a configuration database at startup. Besides this non-real-time communication, realtime communication is also supported out-of-the-box. At the Mercator telescope we will use EtherCAT for all our field-level communication, and IEEE 1588 (the "Precision Time Protocol" or PTP) for time synchronisation. Considering the support for real-time $\mathrm{C}++$, motion, safety and IEEE 1588 synchronisation, a soft-PLC forms an excellent platform for hosting the pointing kernel of the telescope.

The aforementioned Nasmyth mechanism, as well as the MAIA instrument (section 3), are already controlled by such a PLC system, and this will also be the case for the new mirror cover mechanism (Fig. 3). Gradually, all the telescope control components will be upgraded to PLC control and we plan to have the new TCS fully deployed by the end of 2015. These groundbreaking developments will make Mercator the first telescope under complete industrial PLC control. We are convinced that this fully integrated PLC approach is an ideal choice for the telescope and instrument control in many future applications.

\subsection{Scheduling software}

To provide the PIs of the various observing programs with a way to easily submit their technical requirements and keep track of the progress of their observations, we developed dedicated scheduling and archiving software [4]. This tool is especially important for programs with strict sampling requirements, stringent timing requirements, like e.g. eclipse observations and/or multi-site monitoring campaigns. All scientific programmes are merged in a database and the observer schedules the night from the queue of observing blocks. This ensures that all programmes are performed within their specific requirements. We fully integrated this scheduling software in 


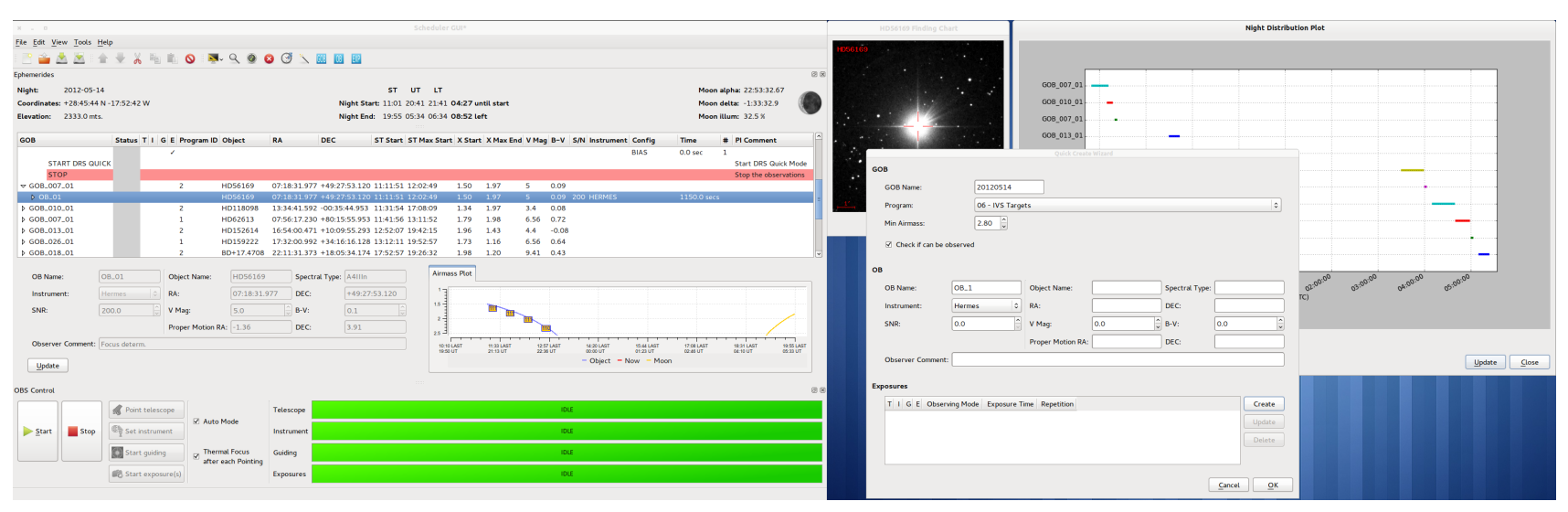

Figure 4. Graphical user interface of the scheduler software.

our new observing system. It allows the observer to create near-optimal real-time observing schedules for each observing night and to execute these schedules in a semi-automatic way (Fig. 4). This makes observing with the Mercator telescope both user friendly and efficient.

\section{INSTRUMENTATION}

Currently, the instrumentation suite of the Mercator telescope consists of only two instruments, the highresolution spectrograph HERMES and the 3-channel imager MAIA. Although more free focal stations are available for additional instruments, no immediate plans for new instrumentation exist. Because of the intensive use of Mercator for monitoring campaigns, there is no interest to disperse the observing time over too many instruments.

\subsection{HERMES}

HERMES is a fibre-fed high-resolution echelle spectrograph with good stability and excellent throughput [5]. After its commissioning in 2009, HERMES immediately became the work-horse instrument of the Mercator telescope, being used for more than $75 \%$ of the available observing time. HERMES mainly targets building up time series of high-quality data of variable stellar phenomena, typically for asteroseismology and binaryevolution research. The layout of HERMES is based on a white pupil design, an R2.7 echelle diffraction grating $(\varnothing 154 \times 408 \mathrm{~mm})$, and two large prisms for cross dispersion (Fig. 5). HERMES stands out by its excellent throughput (up to $28 \%$ ) at a high resolution of $\lambda / \Delta \lambda=85000$, that is achieved thanks to a two-slice image slicer and an efficient optical fibre link ( $\varnothing 80 \mu \mathrm{m}$ or $2.5 \mathrm{arcsec})$. The spectrograph covers the extended optical spectrum from 380 to $900 \mathrm{~nm}$ in a single exposure. To ensure high instrumental stability, it is installed in a chamber with precise environment control. Temperature fluctuations are smaller than $0.01^{\circ} \mathrm{C}$ (long term) or $0.001^{\circ} \mathrm{C}$ (one night) rms. A specific pipeline was developed which yields both fast on-line datareduction for quality control during the observations as well as science quality automatic reduction of all spectra while the observer is sleeping.

Currently, a new and improved fibre link between telescope and spectrograph is under design. This upgrade should further increase the spectrograph throughput and, more importantly, lead to higher accuracy for radial velocity measurements. We plan to use octagonal fibres that have better scrambling properties than standard circular fibres, leading to a more stable illumination of the spectrograph. Moreover, the new fibre link will allow to use the simultaneous Thorium method for tracking any instrumental drift during the exposure, also in high-resolution (HR) mode. For the moment, this way of observing is not possible in HR mode because the cross-order profile of the sliced HR fibre is too wide or cross dispersion too small to fit an additional interlaced spectrum. For that reason, we will install a very narrow fibre $(\varnothing 25 \mu \mathrm{m})$ to feed the wavelength reference source to the spectrograph (Fig. 6). We plan to commission the new set of optical fibres by the end of 2015 . 


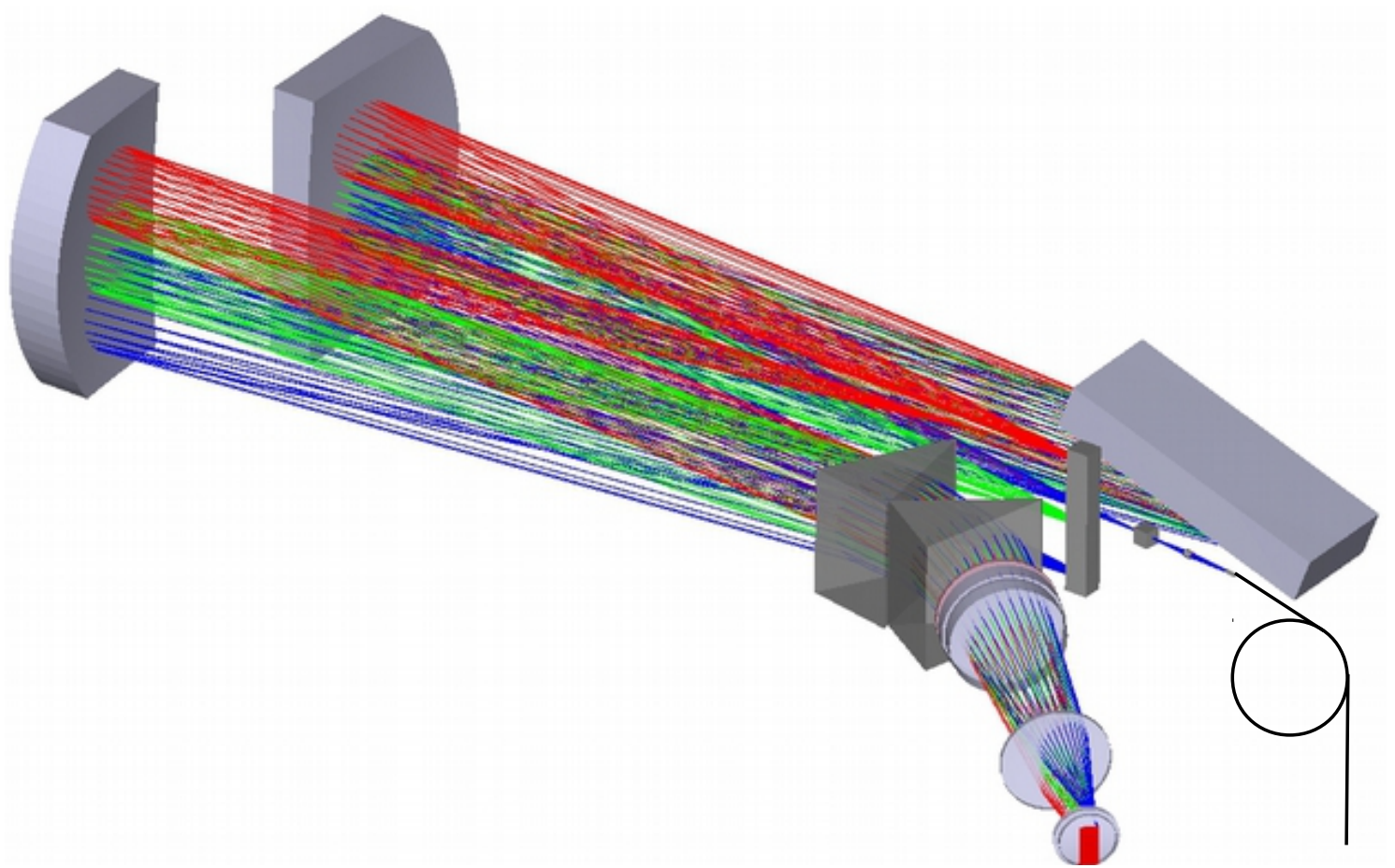

Figure 5. HERMES spectrograph layout.

\subsection{MAIA}

MAIA, an acronym for Mercator Advanced Imager for Asteroseismology, was installed in 2012 to replace the decommissioned MEROPE camera (Fig. 1\& 7) [6]. This instrument is designed to perform fast-cadence threecolour observations for asteroseismology, with specific emphasis on subdwarf and white dwarf single and binary stars. MAIA can observe a 9.4" x 14.1" field-of-view simultaneously in three wavelength bands $(u, g$ and $r)$ on three large $(2 \mathrm{k} \times 6 \mathrm{k})$ frame-transfer CCDs. These detectors, cooled with mechanical Stirling coolers [7], are optimised for rapid read out [8]. They were originally developed for ESA's cancelled Eddington space mission and, after the cancellation of Eddington, offered on permanent loan to the Institute of Astronomy (KU Leuven, Belgium). In case of windowed detector read-out, MAIA can run at a cycle time of only a few seconds with negligible dead time between the exposures. This makes this instrument ideally suited for fast multi-colour photometry. At the same time, MAIA is sufficiently versatile to serve many other applications.

\section{SCIENCE WITH MERCATOR: RELEVANCE AND RESULTS}

The strongest scientific asset of the Mercator telescope lies in its ability to provide stellar astrophysicists with high-quality time series of high-resolution spectra and of simultaneous three-colour precision photometry, and this with a very wide range of sampling times. Mercator is also one of the few telescopes that enables very long monitoring programmes, in some cases extending up to several years, ideal for e.g. asteroseismology and binary star research.

While important asteroseismology missions like CoRot and Kepler recently finished their active science mission, the legacy of these satellites comprises the need for ground-based follow-up with high-resolution spectroscopy during many years to come. Although Kepler's main mission is over, also for the extended mission K2, the Mercator telescope is used to obtain much needed preparatory ground-based support as well as for follow-up spectral time-series. The successful exploitation of the synergy between ground-based and space-borne infrastructure lead already to important advances in our understanding how stars evolve. The application of asteroseismology to a wide range of variable stars to gain insight in their stellar interior will remain an important science goal of 


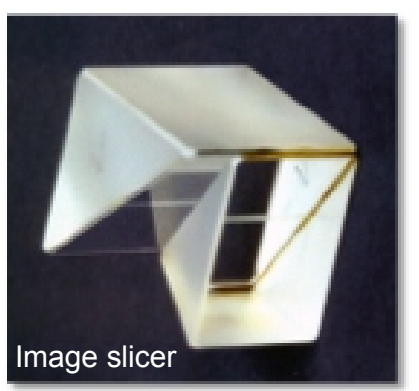

\section{Cross-section side view}

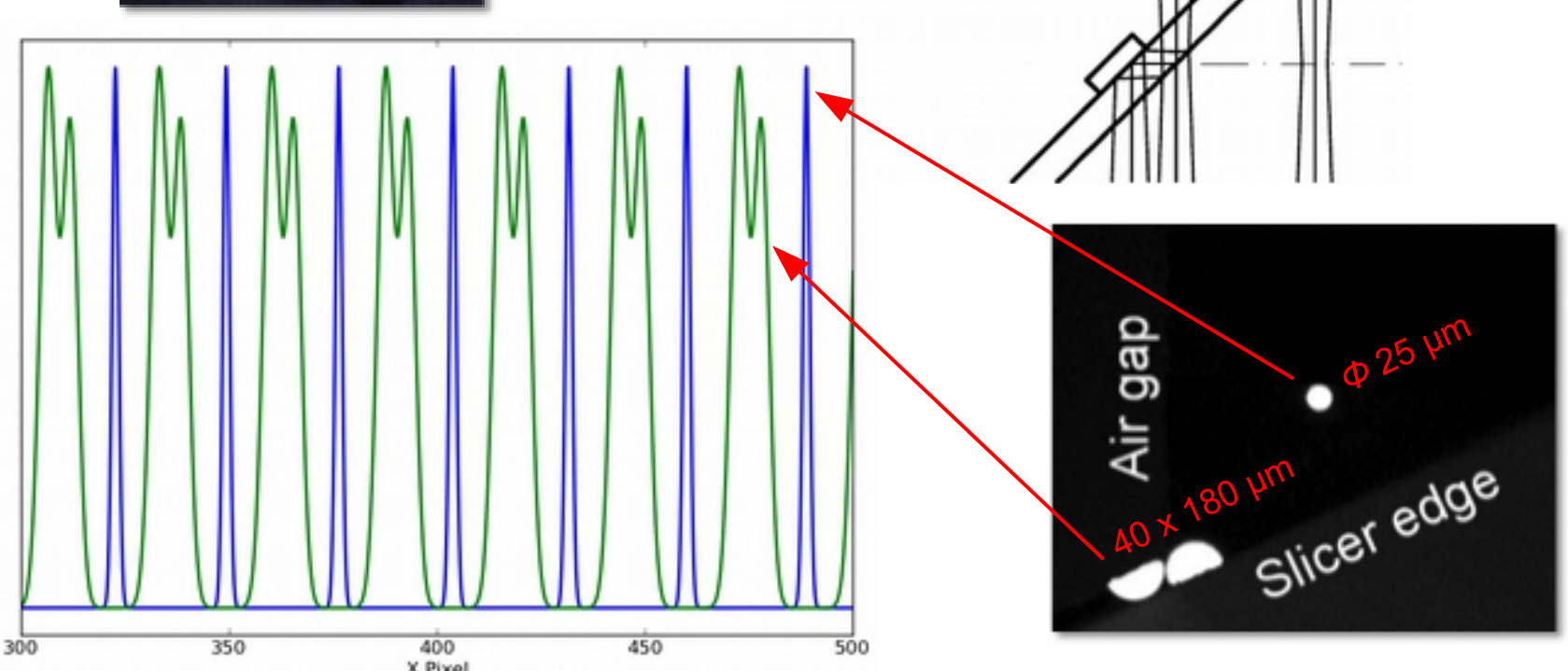

Figure 6. Picture of the HERMES two-slice image slicer (top left); simulation of how the beams from the sliced science and $25-\mu \mathrm{m}$ reference fibres pass through the slicer (right); part of the cross-order profile of science (green) and narrow reference (blue) spectra (bottom left).
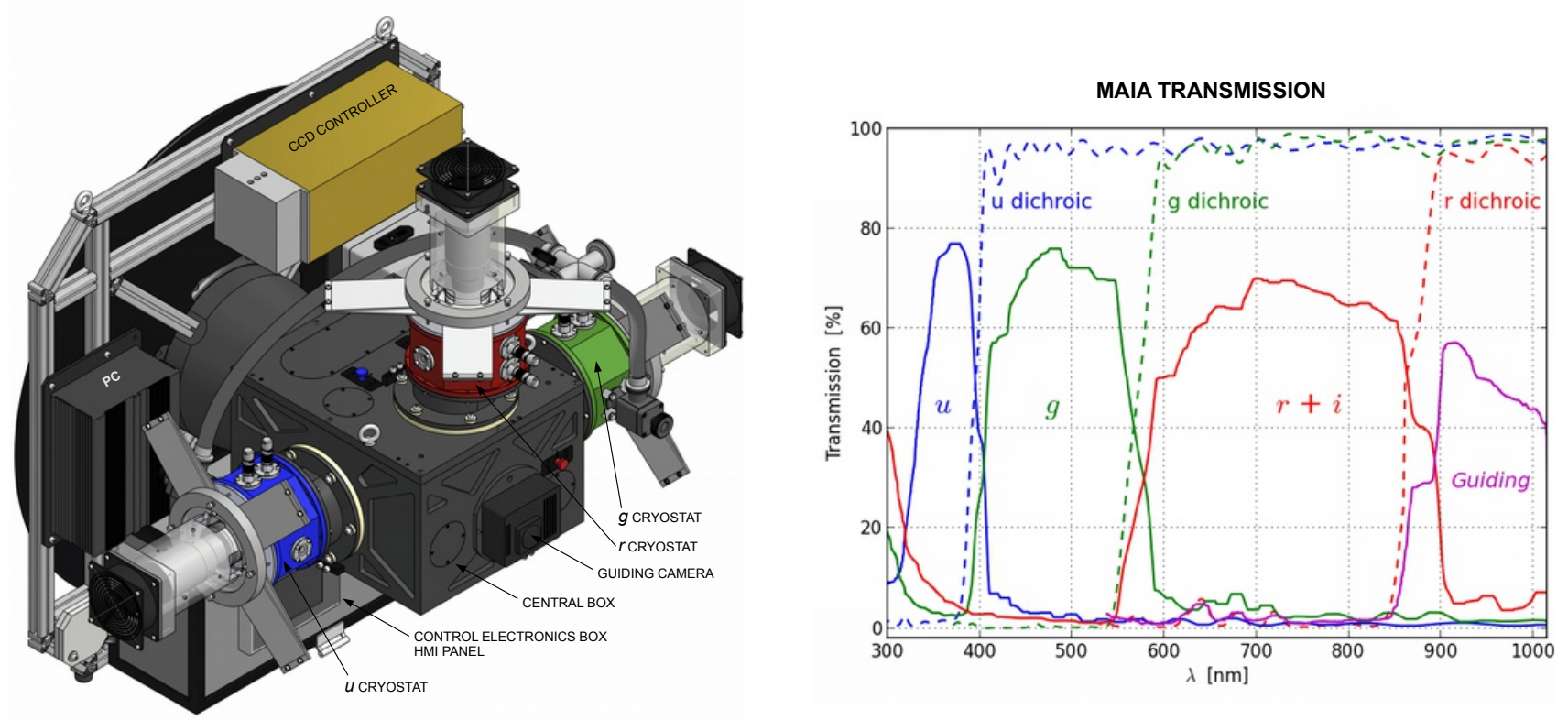

Figure 7. 3D drawing of MAIA with the 3 cryostats in their respective colours (left); spectral transmission of the 3 MAIA channels, detectors or additional filters not included (right). 
Mercator. Also in the future, this will require spectroscopic monitoring programmes with a specific cadence tuned to the variability timescale.

Asteroseismology research based on $\mu$ mag-precise space photometry has resulted in a revolution in our knowledge of stellar interiors. However, some types of pulsating stars could not be studied by missions like MOST, CoRoT or Kepler. This is particularly the case for fast pulsators like roAp stars, pressure-mode pulsating subdwarfs and gravity-mode pulsating white dwarfs. With MAIA on the mercator telescope, asteroseismology of such fast and relatively faint pulsators becomes possible. In contrast to the aforementioned white-light space missions, that enable very accurate detection of the stellar pulsation periods, the multi-colour approach of MAIA will also allow or improve the unambiguous identification of the pulsation modes.

Binary star research, and in particular binary star research of low-to intermediate-mass evolved stars, also requires dedicated spectroscopic monitoring that covers several orbital cycles. As the orbits are often several years long, a continuous effort is required to deepen our understanding of binary evolution. With the results we have obtained so far, we can state already that the circularisation and synchronisation processes of these objects turn out to be much less efficient than what theory predicts: many systems with evolved components are much more eccentric than predicted and this over a wide range of systems. Binary star research requires strong constraints from observations, as many physical processes cannot be fully understood from first principles. This is even more the case when one of the components has evolved. This extensive research to probe binary interaction processes will remain an important scientific goal for the Mercator telescope.

Since the start of science observations with the HERMES spectrograph in April 2009, we have collected more than 45000 science spectra during a total of almost 1450 observing nights. So far, this resulted in more than 140 scientific publications, (partly) based on HERMES data. Highlights of the HERMES exploitation range from the discovery of the extended overshoot of the convective core of a massive B-star [9], deriving the internal rotation profile of the core of a red giant [10], the surprising discovery of long-period eccentric binaries among sub-dwarf B stars [11, 12], the mode identification of single and binary pulsators [13, 14, 15], the discovery of the binary properties of red giant pulsators in the Kepler field and also often in eccentric systems [16, 17], the circumstellar gas flows in binary stars with interacting winds or accretion flows [18, 19], to the first discovery of the long sought wide binaries among central stars of Planetary Nebulae [20]. These are only some illustrative example of science programmes that require the unique monitoring capabilities of HERMES on the Mercator telescope.

\section{CONCLUSION}

The examples of the previous section illustrate that today, with extremely large telescopes dawning at the horizon, a small but dedicated telescope is still capable of producing unique science, provided it is equipped with efficient instruments. Moreover, this can be done at just a fraction of the cost of large facilities. The Mercator telescope, combined with the very efficient HERMES spectrograph or the multi-colour imager MAIA, and with its continuous long-term availability, is a good example of how this can be achieved successfully. Accordingly, the productivity of the Mercator telescope forms a key element in many of the science programs that are conducted at the Institute of Astronomy of the KU Leuven and at the other HERMES consortium partner institutes. To further improve the quality of the observations and to guarantee the long-term maintainability of the facility, we are currently developing an upgraded fibre link for the HERMES spectrograph and a new advanced control system for the telescope.

\section{ACKNOWLEDGMENTS}

The authors acknowledge the financial support from the Fund for Scientific Research of Flanders (FWO) under the Big Science program grants G.0703.08 and G.0C31.13.

\section{References}

[1] G. Raskin, G. Burki, M. Burnet, G. Davignon, R. Dubosson, E. Ischi, M. George, M. Grenon, C. Maire, H. Van Winckel, C. Waelkens, and L. Weber, "Mercator and the P7-2000 photometer," in Society of PhotoOptical Instrumentation Engineers (SPIE) Conference Series, A. F. M. Moorwood and M. Iye, eds., 5492, p. 830 , Sept. 2004. 
[2] G. Raskin, R. Dubosson, B. Michaud, W. Pessemier, and H. Van Winckel, "A new Nasmyth mirror mechanism increases the number of focal stations of the Mercator Telescope," in Society of Photo-Optical Instrumentation Engineers (SPIE) Conference Series, 8446, Sept. 2012.

[3] W. Pessemier, G. Raskin, G. Deconinck, P. Saey, and H. Van Winckel, "Design and first commissioning results of plc-based control systems for the mercator telescope," in Society of Photo-Optical Instrumentation Engineers (SPIE) Conference Series, 8451, 2012.

[4] F. Merges, S. Prins, W. Pessemier, G. Raskin, J. Perez Padilla, H. Van Winckel, and C. Aerts, "MESA: Mercator scheduler and archive system," in Society of Photo-Optical Instrumentation Engineers (SPIE) Conference Series, Society of Photo-Optical Instrumentation Engineers (SPIE) Conference Series 8451, Sept. 2012.

[5] G. Raskin, H. van Winckel, H. Hensberge, A. Jorissen, H. Lehmann, C. Waelkens, G. Avila, J. de Cuyper, P. Degroote, R. Dubosson, L. Dumortier, Y. Frémat, U. Laux, B. Michaud, J. Morren, J. Perez Padilla, W. Pessemier, S. Prins, K. Smolders, S. van Eck, and J. Winkler, "HERMES: a high-resolution fibre-fed spectrograph for the Mercator telescope," A\&A 526, pp. A69+, Feb. 2011.

[6] G. Raskin, S. Bloemen, J. Morren, J. Perez Padilla, S. Prins, W. Pessemier, J. Vandersteen, F. Merges, R. Østensen, H. Van Winckel, and C. Aerts, "MAIA, a three-channel imager for asteroseismology: instrument design," A\&A 559, p. A26, Nov. 2013.

[7] G. Raskin, J. Morren, W. Pessemier, J. Perez Padilla, and J. Vandersteen, "Compact Stirling cooling of astronomical detectors," ArXiv e-prints , Nov. 2013.

[8] J. Pérez Padilla, S. Tulloch, G. Raskin, S. Prins, F. Merges, W. Pessemier, and S. Bloemen, "Large FrameTransfer Detectors for the MAIA Imager," ArXiv e-prints, Nov. 2013.

[9] P. Degroote, C. Aerts, A. Baglin, A. Miglio, M. Briquet, A. Noels, E. Niemczura, J. Montalban, S. Bloemen, R. Oreiro, M. Vučković, K. Smolders, M. Auvergne, F. Baudin, C. Catala, and E. Michel, "Deviations from a uniform period spacing of gravity modes in a massive star," Nature 464, pp. 259-261, Mar. 2010.

[10] P. G. Beck, J. Montalban, T. Kallinger, J. De Ridder, C. Aerts, R. A. García, S. Hekker, M.-A. Dupret, B. Mosser, P. Eggenberger, D. Stello, Y. Elsworth, S. Frandsen, F. Carrier, M. Hillen, M. Gruberbauer, J. Christensen-Dalsgaard, A. Miglio, M. Valentini, T. R. Bedding, H. Kjeldsen, F. R. Girouard, J. R. Hall, and K. A. Ibrahim, "Fast core rotation in red-giant stars as revealed by gravity-dominated mixed modes," Nature 481, pp. 55-57, Jan. 2012.

[11] J. Vos, R. H. Østensen, P. Németh, E. M. Green, U. Heber, and H. Van Winckel, "The orbits of subdwarf-B + main-sequence binaries. II. Three eccentric systems; BD $+29^{\mathrm{deg}} 3070$, BD $+34^{\mathrm{deg}} 1543$ and Feige 87," $A \& A$ 559, p. A54, Nov. 2013.

[12] R. H. Østensen and H. Van Winckel, "Radial-velocity Monitoring of Long-period Hot-subdwarf + Mainsequence Binaries with HERMES@Mercator," in Fifth Meeting on Hot Subdwarf Stars and Related Objects, D. Kilkenny, C. S. Jeffery, and C. Koen, eds., Astronomical Society of the Pacific Conference Series 452, p. 163, Mar. 2012.

[13] P. Degroote, C. Aerts, E. Michel, M. Briquet, P. I. Pápics, P. Amado, P. Mathias, E. Poretti, M. Rainer, R. Lombaert, M. Hillen, T. Morel, M. Auvergne, A. Baglin, F. Baudin, C. Catala, and R. Samadi, "The CoRoT B-type binary HD 50230: a prototypical hybrid pulsator with g-mode period and p-mode frequency spacings," A\&A 542, p. A88, June 2012.

[14] J. Debosscher, C. Aerts, A. Tkachenko, K. Pavlovski, C. Maceroni, D. Kurtz, P. G. Beck, S. Bloemen, P. Degroote, R. Lombaert, and J. Southworth, "KIC 11285625: A double-lined spectroscopic binary with a $\gamma$ Doradus pulsator discovered from Kepler space photometry," A\&GA 556, p. A56, Aug. 2013. 
[15] A. Tkachenko, C. Aerts, A. Yakushechkin, J. Debosscher, P. Degroote, S. Bloemen, P. I. Pápics, B. L. de Vries, R. Lombaert, M. Hrudkova, Y. Frémat, G. Raskin, and H. Van Winckel, "Detection of a large sample of $\gamma$ Doradus stars from Kepler space photometry and high-resolution ground-based spectroscopy," $A \mathscr{E} A$ 556, p. A52, Aug. 2013.

[16] S. Frandsen, H. Lehmann, S. Hekker, J. Southworth, J. Debosscher, P. Beck, M. Hartmann, A. Pigulski, G. Kopacki, Z. Kołaczkowski, M. Stȩślicki, A. O. Thygesen, K. Brogaard, and Y. Elsworth, "KIC 8410637: a 408-day period eclipsing binary containing a pulsating giant star," A $6 A$ 556, p. A138, Aug. 2013.

[17] P. G. Beck, K. Hambleton, J. Vos, T. Kallinger, S. Bloemen, A. Tkachenko, R. A. García, R. H. Østensen, C. Aerts, D. W. Kurtz, J. De Ridder, S. Hekker, K. Pavlovski, S. Mathur, K. De Smedt, A. Derekas, E. Corsaro, B. Mosser, H. Van Winckel, D. Huber, P. Degroote, G. R. Davies, A. Prša, J. Debosscher, Y. Elsworth, P. Nemeth, L. Siess, V. S. Schmid, P. I. Pápics, B. L. de Vries, A. J. van Marle, P. MarcosArenal, and A. Lobel, "Pulsating red giant stars in eccentric binary systems discovered from Kepler spacebased photometry. A sample study and the analysis of KIC 5006817," A\&A 564, p. A36, Apr. 2014.

[18] N. Gorlova, H. Van Winckel, C. Gielen, G. Raskin, S. Prins, W. Pessemier, C. Waelkens, Y. Frémat, H. Hensberge, L. Dumortier, A. Jorissen, and S. Van Eck, "Time-resolved spectroscopy of BD+46 ${ }^{\text {deg }} 442$ : Gas streams and jet creation in a newly discovered evolved binary with a disk," $A \& A$ 542, p. A27, June 2012.

[19] A. Lobel, J. H. Groh, C. Martayan, Y. Frémat, K. Torres Dozinel, G. Raskin, H. Van Winckel, S. Prins, W. Pessemier, C. Waelkens, H. Hensberge, L. Dumortier, A. Jorissen, S. Van Eck, and H. Lehmann, "Modelling the asymmetric wind of the luminous blue variable binary MWC 314," $A \mathscr{G} A$ 559, p. A16, Nov. 2013 .

[20] H. Van Winckel, A. Jorissen, K. Exter, G. Raskin, S. Prins, J. Perez Padilla, F. Merges, and W. Pessemier, "Binary central stars of planetary nebulae with long orbits: the radial velocity orbit of BD $+33^{\text {deg }} 2642(\mathrm{PN}$ G052.7+50.7) and the orbital motion of HD 112313 (PN LoTr5)," A $6 A$ 563, p. L10, Mar. 2014. 